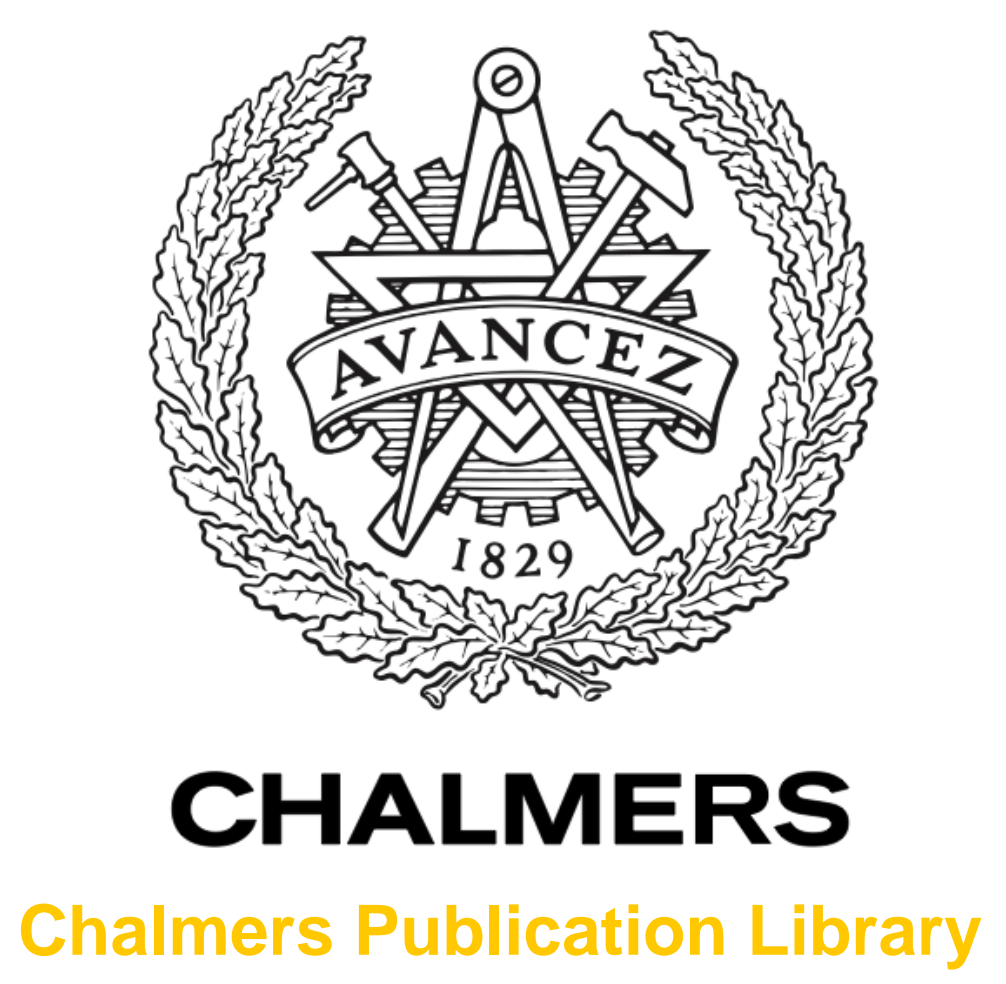

\title{
Spin transport and precession in graphene measured by nonlocal and three-terminal methods
}

This document has been downloaded from Chalmers Publication Library (CPL). It is the author's version of a work that was accepted for publication in:

Applied Physics Letters (ISSN: 0003-6951)

Citation for the published paper:

Dankert, A. ; Mutta, V. ; Dash, S. (2014) "Spin transport and precession in graphene measured by nonlocal and three-terminal methods". Applied Physics Letters, vol. 104(19), pp. 192403.

http://dx.doi.org/10.1063/1.4876060

Downloaded from: http://publications.lib.chalmers.se/publication/198050

Notice: Changes introduced as a result of publishing processes such as copy-editing and formatting may not be reflected in this document. For a definitive version of this work, please refer to the published source. Please note that access to the published version might require a subscription.

Chalmers Publication Library (CPL) offers the possibility of retrieving research publications produced at Chalmers University of Technology. It covers all types of publications: articles, dissertations, licentiate theses, masters theses, conference papers, reports etc. Since 2006 it is the official tool for Chalmers official publication statistics. To ensure that Chalmers research results are disseminated as widely as possible, an Open Access Policy has been adopted.

The CPL service is administrated and maintained by Chalmers Library. 


\title{
Spin transport and precession in graphene measured by nonlocal and three-terminal methods
}

\author{
André Dankert, $\left.{ }^{a}\right)$ Mutta Venkata Kamalakar, Johan Bergsten, and Saroj P. Dash ${ }^{\text {b) }}$ \\ Department of Microtechnology and Nanoscience, Chalmers University of Technology, SE-41296 Göteborg, \\ Sweden
}

(Received 11 March 2014; accepted 30 April 2014; published online 13 May 2014)

\begin{abstract}
We investigate the spin transport and precession in graphene by using the Hanle effect in nonlocal and three-terminal measurement geometries. Identical spin lifetimes, spin diffusion lengths, and spin polarizations are observed in graphene devices for both techniques over a wide range of temperatures. The magnitude of the spin signals is well explained by spin transport models. These observations rules out any signal enhancements or additional scattering mechanisms at the interfaces for both geometries. This validates the applicability of both the measurement methods for graphene based spintronics devices and their reliable extractions of spin parameters. (C) 2014 AIP Publishing LLC. [http://dx.doi.org/10.1063/1.4876060]
\end{abstract}

The spin degree of freedom of electrons is considered as an alternative state variable for processing information beyond the charge based CMOS technology. Its potential lies in the possibilities for a new generation of computers that can be non-volatile, faster, smaller, and capable of simultaneous data storage and processing with reduced energy consumption. ${ }^{1}$ The strong interest in graphene and silicon based spintronic devices stems from their potentially long spin coherence lengths due to the absence of hyperfine interactions and a weak spin-orbit coupling. Such materials could be employed in the recently proposed concept of all-spin logic using spins in ferromagnets to store information and communicate between them using a spin current. ${ }^{2}$ All-spin logic is particularly powerful since it combines various spin related phenomena such as spin injection, transport, and detection with magnetization dynamics.

In order to achieve these goals, various methods for electrical spin injection and detection in metals, ${ }^{3}$ semiconductors, ${ }^{4-6}$ and graphene ${ }^{7}$ have been investigated. Primarily nonlocal (NL) and three-terminal (3T) methods are used for an electrical detection of the spin polarization. ${ }^{6-9}$ The nonlocal geometry separates the current and voltage path to provide information about pure spin transport parameters. However, nanofabrication by electron beam lithography is necessary in order to achieve submicrometer structures and channel lengths. ${ }^{7}$ Although the NL method has been widely used for spin transport measurements in more conducting metals, ${ }^{3}$ graphene, ${ }^{7}$ and $\mathrm{GaAs},{ }^{4,10}$ it has been found to be challenging for $\mathrm{Si}$ and $\mathrm{Ge}$ based devices due to the high resistive Schottky barriers. Nevertheless, there are few reports studying NL signals in those materials at low temperature, ${ }^{11}$ and more recently even at room temperature. $^{12-14}$ Therefore, the $3 \mathrm{~T}$ Hanle technique was preferred for measuring spin signals in semiconductor materials, since it allows to study the creation and detection of spin accumulations by a single magnetic tunnel contact up to room temperature in a reproducible way. $6,8,15$

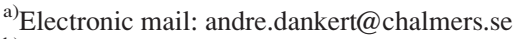

b)Electronic mail: saroj.dash@chalmers.se
}

However, there has been a continuing discussion about spin parameters obtained by the $3 \mathrm{~T}$ technique. On the one hand, it has been reported that the $3 \mathrm{~T}$ geometry provides a lower limit for spin lifetimes and larger magnitudes of the spin signal in semiconductors than theoretically expected. ${ }^{6,8,16}$ On the other hand, spin lifetimes in metals obtained by $3 \mathrm{~T}$ measurements are significantly longer than theoretically predicted. ${ }^{17}$ Several experiments also proposed an enhancement $^{15,18}$ or inversion ${ }^{8}$ of the $3 \mathrm{~T}$ spin signal, whereas multiple control experiments rule out any enhancement due to interface states. ${ }^{6,16,19}$ Therefore, it is required to understand the contributing factors in both techniques by performing experiments in the NL and 3T configuration using the same magnetic tunnel contacts. Such measurements have been demonstrated for $\mathrm{Si}^{11,12,14} \mathrm{GaAs}^{20}$ and epitaxial graphene on $\mathrm{SiC}$ substrate ${ }^{21}$ at temperatures up to $100 \mathrm{~K}$, and on $\mathrm{Ge}^{13}$ up to room temperature. Even though there is a good agreement between the NL and $3 \mathrm{~T}$ spin parameters for most of those cases, some deviations were observed for $\mathrm{Ge}$ at higher temperatures. This has been explained by the fact that the 3T Hanle measurement is more easily affected by additional scattering effects caused by the accompanied charge current and the electric field under the FM contact. In spite of the importance of studying those effects, especially at higher temperatures, none of the other articles compares data above $100 \mathrm{~K}$. Although, spin transport in exfoliated graphene has been studied the most, measurements on different geometries have not been reported yet.

Here, we present spin transport and precession measurements in the same exfoliated graphene device using both NL and $3 \mathrm{~T}$ measurement geometries. By analyzing the data on the basis of spin diffusion model, we show a very good agreement between the parameters obtained from both methods up to room temperature. This indicates that there are no additional enhancement or scattering mechanisms for the $3 \mathrm{~T}$ geometry and validates the applicability of both methods for spin transport and precession measurements in graphene.

The graphene flakes were exfoliated from highly oriented pyrolytic graphite (advanced ceramics), using the conventional cleavage technique, onto a clean $\mathrm{SiO}_{2}$ $(285 \mathrm{~nm}) /$ highly doped n-type $\mathrm{Si}$ substrate. The flake's 
(a)
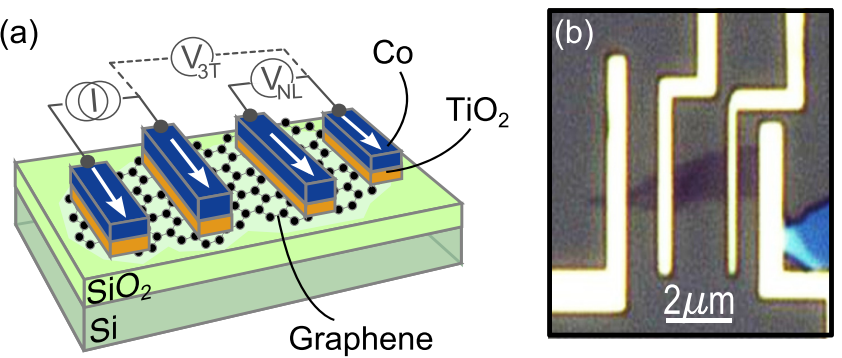

FIG. 1. Graphene spintronic device and measurement geometry. (a) Schematic representation the graphene spintronic device with ferromagnetic tunnel contacts on $\mathrm{SiO}_{2} / \mathrm{Si}$ substrate for a $\mathrm{NL}$ and $3 \mathrm{~T}$ configuration. (b) Optical microscope image of a multi-terminal spin-transport device showing a graphene flake contacted by $\mathrm{TiO}_{2}(1 \mathrm{~nm}) / \mathrm{Co} / \mathrm{Au}$ electrodes patterned by electron beam lithography.

thickness was characterized using a combination of optical and atomic-force microscopy. In our experiments, we used graphene flakes with a thickness of 2-3 layers, and a widths of around $W=1.6 \mu \mathrm{m}$. Ferromagnetic electrodes of different widths $(0.2-1 \mu \mathrm{m})$ for the spin injector and detector were fabricated by electron beam lithography, electron beam deposition and lift-off technique. The shape anisotropy ensures different switching fields of the electrodes allowing parallel and antiparallel configurations through in-plane magnetic field sweeps. The injector and detector electrodes were placed in a distance $L=2 \mu \mathrm{m}$. The contacts consist of $1 \mathrm{~nm}$ $\mathrm{TiO}_{2}$ tunnel barrier, $65 \mathrm{~nm} \mathrm{Co}$, and $20 \mathrm{~nm}$ Au capping layer. The $\mathrm{TiO}_{2}$ barrier was prepared by a twofold evaporation of $5 \AA$ of Ti and oxidation in an oxygen atmosphere. This ensures a homogenous and fully oxidized metaloxide barrier.

The electrical characterization of the device was performed using a multi-terminal measurement geometry (Fig. 1). The contact resistance is about $R_{c} \approx 2.3 \mathrm{k} \Omega\left(R_{c} A \approx\right.$ $600 \Omega \mu \mathrm{m}^{2}$ ) at $290 \mathrm{~K}$ with a nonmetallic temperature dependence indicating a pinhole free tunnel barrier. ${ }^{22}$ The channel resistivity of the graphene was found to be $R_{\square} \approx 1 \mathrm{k} \Omega$. Since $R_{c}>R_{\square}$, the back-flow of the injected spins into the FM should be effectively suppressed. ${ }^{23}$ The graphene channel showed a regular gate-dependent Dirac curve with a mobility $\mu \approx 2500 \mathrm{~cm}^{2}(\mathrm{~V} \mathrm{~s})^{-1}$. Spin transport measurements were performed in both NL and 3T geometry using a direct current (DC), whereas the voltage was detected by a nanovoltmeter (Fig. 1(a)). The measurements were carried out under vacuum in a variable temperature cryostat with a superconducting magnet.

The spin transport was studied in the nonlocal spinvalve geometry, where the charge current path is isolated from the spin diffusion. The spins injected through the $\mathrm{Co} / \mathrm{TiO}_{2}$ contacts accumulate in the graphene, diffuse laterally and get detected by the nonlocal voltage probes (inset of Fig. 2). The nonlocal resistance is recorded while the in-plane magnetic field is swept from a negative to a positive value, followed by a reverse sweep. A nonlocal measurement performed with a DC $I=5 \mu \mathrm{A}$, at room temperature is shown in Fig. 2. A distinct spin-valve switching has been observed between the parallel and antiparallel configurations of the injector and detector electrodes, with a spin signal of $R_{N L}=110 \mathrm{~m} \Omega$. This demonstrates the spin injection, transport, and detection in our graphene device.

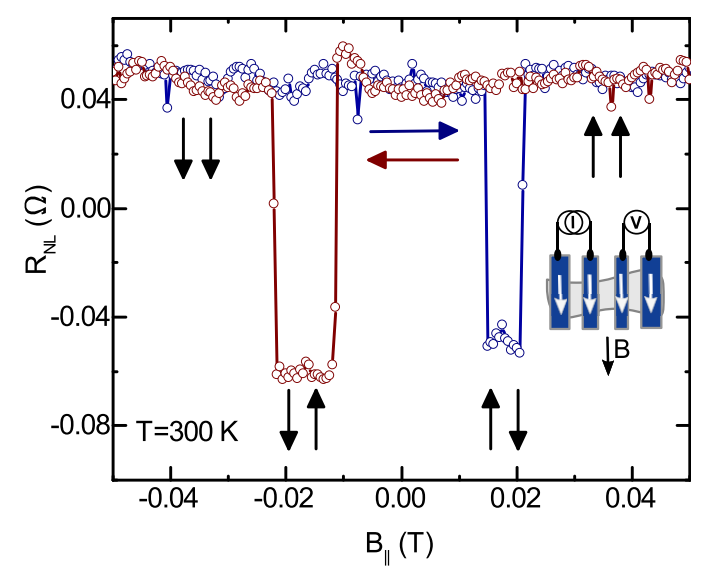

FIG. 2. Nonlocal spin-valve measurement. Nonlocal spin-valve signal measured at $300 \mathrm{~K}$ using an injection current of $I=5 \mu \mathrm{A}$. The sweep directions of the in-plane magnetic field are indicated by blue (trace) and red (retrace). The magnetic configurations of the electrodes are illustrated for both sweep directions.

In order to evaluate the spin lifetime of the electrons in the graphene device, we performed Hanle spin precession measurements in the NL and 3T geometry. The NL Hanle spin precession measurement is performed by sweeping the magnetic field perpendicular to the device geometry, with the magnetization axes of the injector and detector electrodes kept parallel (inset Fig. 3(a)). The injected spin-polarized electrons precess around the perpendicular magnetic field $B_{\perp}$

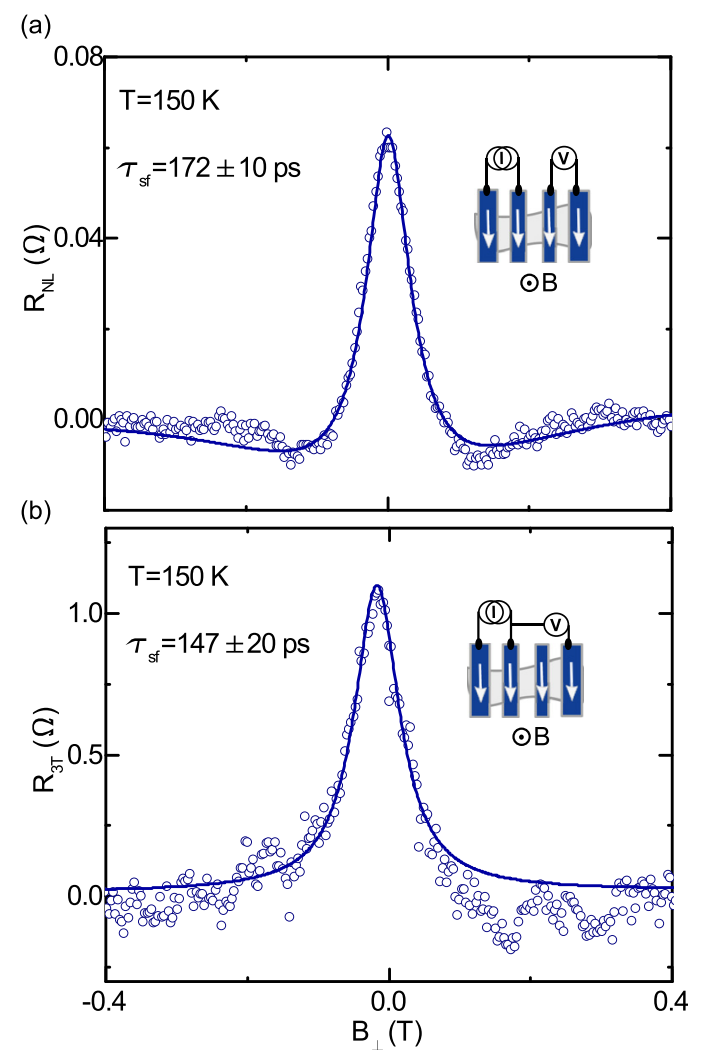

FIG. 3. Hanle measurements in NL and 3T geometry. (a) NL Hanle spinprecession signal performed with an injection current of $I=5 \mu \mathrm{A}$ at $150 \mathrm{~K}$. The solid line represents a nonlocal Hanle fit with spin drift, diffusion, and precession equation as in Eq. (1) with a spin lifetime $\tau_{s f}=172 \pm 10 \mathrm{ps}$ and $D=0.008 \mathrm{~m}^{2} \mathrm{~s}^{-1}$. (b) $3 \mathrm{~T}$ Hanle signal performed with an injection current of $I=5 \mu \mathrm{A}$ at $150 \mathrm{~K}$. The solid line is the Lorentzian fit for 3T Hanle data as presented in Eq. (2). 
with the Larmor frequency $\omega_{\mathrm{L}}=g \mu_{\mathrm{B}} B_{\perp} \hbar^{-1}$ (Lande's gfactor $g=2$ ), while diffusing towards the nonlocal detector contact. The variation of this nonlocal resistance $\left(\Delta R_{N L}\right)$ due to precession and relaxation of the spins diffusing from the injector to the detector can be described by

$R_{N L}= \pm \frac{P^{2} R_{\square}}{W} \int_{0}^{\infty} \sqrt{\frac{D}{4 \pi t}} \exp \left[-\frac{L^{2}}{4 D t}\right] \cos \left[\omega_{L} t\right] \exp \left[-\frac{t}{\tau_{s f}}\right] \mathrm{d} t$.

With the measured sheet resistance $R_{\square}$ and predefined channel width $W$ and length $L$, we can extract the spin polarization $P$ of the $\mathrm{Co} / \mathrm{TiO}_{2}$ contact, the diffusion constant $D$, and the spin lifetime $\tau_{s f}$.

Figure 3(a) shows a nonlocal Hanle signal with a DC injection current $I=5 \mu \mathrm{A}$ at $T=150 \mathrm{~K}$. Fitting the data with Eq. (1), we obtain a spin lifetime $\tau_{s f}=172 \pm 10$ ps and a diffusion constant $D=0.008 \mathrm{~m}^{2} \mathrm{~s}^{-1}$ resulting in a spin diffusion length $\lambda_{s f}=\sqrt{D \tau_{s f}}=0.8 \mu \mathrm{m}$, with a spin polarization $P=7 \%$. The observed spin lifetime and diffusion length are significantly shorter than theoretically expected, ${ }^{24}$ but compare well with the reported values for graphene devices on $\mathrm{SiO}_{2}$ using other oxide tunnel barriers ${ }^{7}$ with a similar carrier mobility of $\mu \approx 2500 \mathrm{~cm}^{2}(\mathrm{Vs})^{-1}$.

Next, we focus on Hanle measurements in the $3 \mathrm{~T}$ configuration. The $3 \mathrm{~T}$ configuration is an extreme of a NL measurement scheme in which the distance $L$ between injector and detector becomes zero. That means, the same FM contact is used for spin injection and detection in the graphene. The Hanle effect is used to control the reduction of the induced spin accumulation by precession around an external perpendicular magnetic field $\left(B_{\perp}\right)$. The spin accumulation decays as a function of $B_{\perp}$ with an approximately Lorentzian line shape given by

$$
R_{3 T}=\frac{P^{2} R_{\square} \lambda_{s f}}{2 W\left[1+\left(\omega_{L} \tau_{s f}\right)^{2}\right]} .
$$

Figure 3(b) shows a 3T electrical Hanle signal obtained in the same $\mathrm{Co} / \mathrm{TiO}_{2} /$ graphene contact as used for the NL measurement. At a temperature of $150 \mathrm{~K}$ and a constant tunnel current of $I=5 \mu \mathrm{A}$, the spin resistance is found to be $R_{3 T}=1$ $\Omega$, with a polarization $P=8.1 \%$, and a spin lifetime $\tau_{s f}=147 \pm 20 \mathrm{ps}$. This demonstrates that both NL and 3T measurements show a comparable spin lifetime and polarization in our graphene device.

In order to understand the mechanisms affecting the spin accumulation in both configurations, we compare NL and $3 \mathrm{~T}$ spin parameters at different temperatures (Figure 4). The spin lifetime is found to be identical in the studied temperature range from 50 to $290 \mathrm{~K}$ (Fig. 4(a)). Noticeably, the lifetime significantly decreases for temperatures above $150 \mathrm{~K}$. This has been observed previously in $\mathrm{Si}^{11}$ and $\mathrm{Ge}^{13}$ and could be attributed to phonon induced scattering. In the case of graphene, such a distinct behavior has been previously reported by studying the temperature dependence of the mobility in single and few layer graphene devices. ${ }^{25}$ Above a temperature of $100-200 \mathrm{~K}$ the mobility was also strongly limited by impurities in the graphene itself and
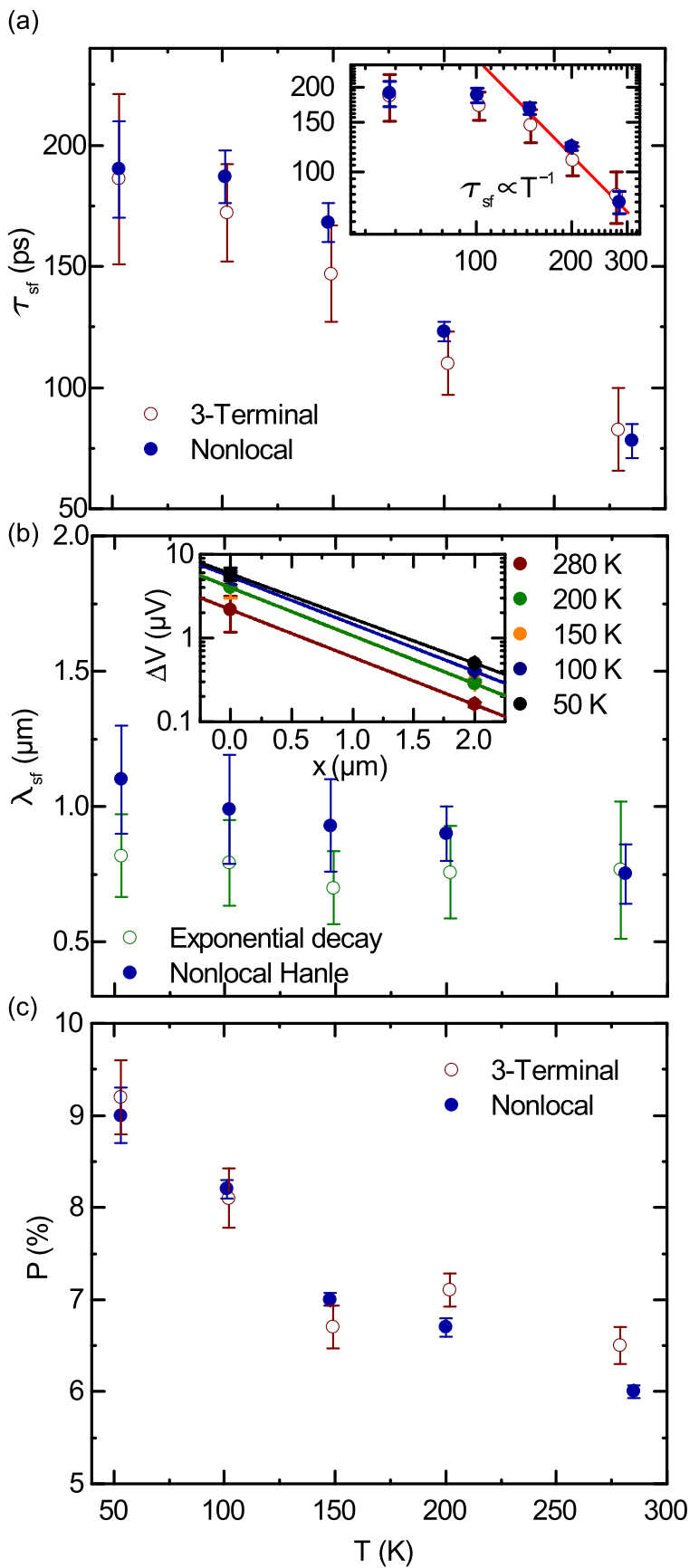

FIG. 4. Comparison of spin parameters for nonlocal and 3-terminal Hanle. (a) Spin lifetimes extracted directly from Hanle fits according to Eqs. (1) and (2). Inset: $\log -\log$ dependence of the main plot indicating a $\tau_{s f} \propto T^{\alpha}$ dependence (red line) with $\alpha=-1 \pm 0.1$ for $T \geq 150 \mathrm{~K}$. (b) Spin diffusion length, as extracted from the nonlocal Hanle fit, compared to the exponential decay of the spin signal amplitude (inset) from the injector to the detector electrode. (c) Spin polarization extracted directly from Hanle fits according to Eqs. (1) and (2).

significantly below the limit of longitudinal acoustic and polar optical phonons of the $\mathrm{SiO}_{2}$ substrate. Assuming $\tau_{s f} \propto T^{\alpha}$, a power-law dependence of the spin lifetime on the temperature, ${ }^{13}$ we extracted a coefficient $\alpha=-1 \pm 0.1$ for temperatures $T \geq 150 \mathrm{~K}$. In contrast to the results observed in $\mathrm{Ge},{ }^{13}$ our matching spin lifetimes, at low as well as for high temperatures, indicate that we have no additional scattering mechanism in neither the $3 \mathrm{~T}$ nor the NL technique. It has to be mentioned that previous temperature dependent studies on single-layer (SLG) and bi-layer (BLG) graphene reported significantly higher spin lifetimes for low 
temperatures with different decays when warming up to room temperature. ${ }^{26}$ The difference in temperature dependence is proposed to stem from a drastic change in the scattering mechanism going from SLG and BLG. Those studies used $\mathrm{TiO}_{2}$ seeded $\mathrm{MgO}$ tunnel barriers. In contrast, our values for the spin lifetime correspond very well with other studies on regular metaloxide tunnel barriers ${ }^{7}$ and are similar to the temperature dependence of $\mathrm{BLG}$ with a $\mathrm{TiO}_{2} / \mathrm{MgO}$ tunnel barrier. ${ }^{26}$ However, the scattering mechanism and spin lifetime can be also affected by contact induced relaxation. ${ }^{23}$

The amplitude of the spin signal, given as

$$
R(x)=\frac{P^{2} R_{\square} \lambda_{s f}}{2 W} \exp \left(-\frac{L}{\lambda_{s f}}\right),
$$

depends exponentially on the distance $L$ between the injector and detector electrode. If the measurement current and detected polarisation are identical in both techniques, Eq. (3) is simplified to $V(x)=V(0) \cdot \exp \left(-L \lambda_{s f}^{-1}\right)$. Indeed, we can show that the polarizations extracted from the $3 \mathrm{~T}$ and NL Hanle measurement are identical (Fig. 4(c)). Employing the simplified Eq. (3), we extracted the spin diffusion length $\lambda_{s f}$ (inset Fig. 4(b)), which is identical to the value extracted from the NL fit with Eq. (1). This confirms that the observed spin signal amplitude of the $3 \mathrm{~T}$ measurement correlates well to the signal of the NL method and rules out any form of spin signal enhancement due to interface effects. ${ }^{8}$

In conclusion, we experimentally demonstrated the spin transport and precession in graphene by studying the Hanle effect in NL and 3T measurement geometries. We observe identical spin lifetimes, diffusion length, and polarization over a wide temperature range. The spin lifetime decays from about $180 \mathrm{ps}$ to $80 \mathrm{ps}$, with a power-law dependence for temperatures above $150 \mathrm{~K}$. The matching lifetimes from both techniques rule out any additional scattering mechanisms for either of the techniques. Furthermore, we were able to demonstrate that the magnitude of the spin signal follows an exponential decay with distance, as shown by the 3T and NL measurements. This rules out any spin signal enhancement due to interface effects in the 3T configuration. This verifies the applicability of both methods for spin transport and precession measurements in graphene allowing for faster developing and studying of future devices and geometries. Furthermore, these results are of great significance for evaluating spin parameters in other semiconducting two-dimensional materials. ${ }^{27}$

The authors acknowledge the support of colleagues at the Quantum Device Physics Laboratory and
Nanofabrication Laboratory at Chalmers University of Technology. The authors would also like to acknowledge the financial supported from the Nano Area of the Advance program at Chalmers University of Technology.

${ }^{1}$ D. Awschalom and M. Flatté, Nature Phys. 3, 153 (2007).

${ }^{2}$ H. Dery, P. Dalal, L. Cywinski, and L. J. Sham, Nature 447, 573 (2007).

${ }^{3}$ F. Jedema, A. T. Filip, and B. J. V. Wees, Nature 410, 345 (2002).

${ }^{4}$ X. Lou, C. Adelmann, S. A. Crooker, E. S. Garlid, J. Zhang, K. S. M. Reddy, S. D. Flexner, C. J. Palmstrøm, and P. A. Crowell, Nature Phys. 3, 197 (2007).

${ }^{5}$ O. M. J. van't Erve, A. T. Hanbicki, M. Holub, C. H. Li, C. Awo-Affouda, P. E. Thompson, and B. T. Jonker, Appl. Phys. Lett. 91, 212109 (2007).

${ }^{6}$ S. P. Dash, S. Sharma, R. S. Patel, M. P. de Jong, and R. Jansen, Nature 462, 491 (2009).

${ }^{7}$ N. Tombros, C. Jozsa, M. Popinciuc, H. T. Jonkman, and B. J. van Wees, Nature 448, 571 (2007).

${ }^{8}$ A. Dankert, R. S. Dulal, and S. P. Dash, Sci. Rep. 3, 3196 (2013).

${ }_{9}^{9}$ A. Dankert and S. P. Dash, Appl. Phys. Lett. 103, 242405 (2013).

${ }^{10}$ G. Salis, S. F. Alvarado, and A. Fuhrer, Phys. Rev. B 84, 041307 (2011).

${ }^{11}$ T. Sasaki, T. Oikawa, M. Shiraishi, Y. Suzuki, and K. Noguchi, Appl. Phys. Lett. 98, 012508 (2011).

${ }^{12}$ T. Suzuki, T. Sasaki, T. Oikawa, M. Shiraishi, Y. Suzuki, and K. Noguchi, Appl. Phys. Express 4, 023003 (2011).

${ }^{13}$ L.-T. Chang, W. Han, Y. Zhou, J. Tang, I. A. Fischer, M. Oehme, J. Schulze, R. K. Kawakami, and K. L. Wang, Semicond. Sci. Technol. 28, 015018 (2013).

${ }^{14}$ T. Sasaki, T. Suzuki, Y. Ando, and H. Koike, e-print arXiv:1401.1279.

${ }^{15}$ A. Jain, M. Cubukcu, J. Peiro, J.-C. Le Breton, E. Prestat, C. Vergnaud, L. Louahadj, C. Portemont, C. Ducruet, V. Baltz, A. Barski, L. Vila, E. Augendre, G. Desfonds, S. Gambarelli, M. Jamet, J.-C. Rojas-Sanchez, P. Bayle-Guillemaud, J.-P. Attané, H. Jaffrès, and J.-M. George, Phys. Rev. Lett. 109, 106603 (2012).

${ }^{16}$ R. Jansen, S. P. Dash, S. Sharma, and B. C. Min, Semicond. Sci. Technol. 27, 083001 (2012).

${ }^{17}$ O. Txoperena, M. Gobbi, A. Bedoya-Pinto, F. Golmar, X. Sun, L. E. Hueso, and F. Casanova, Appl. Phys. Lett. 102, 192406 (2013).

${ }^{18}$ M. Tran, H. Jaffrès, C. Deranlot, J.-M. George, A. Fert, A. Miard, and A. Lemaitre, Phys. Rev. Lett. 102, 036601 (2009).

${ }^{19}$ S. Sharma, A. Spiesser, S. P. Dash, S. Iba, S. Watanabe, B. J. van Wees, H. Saito, S. Yuasa, and R. Jansen, Phys. Rev. B 89, 075301 (2014).

${ }^{20} \mathrm{P}$. Bruski, Y. Manzke, R. Farshchi, O. Brandt, J. Herfort, and M. Ramsteiner, Appl. Phys. Lett. 103, 052406 (2013).

${ }^{21}$ B. Birkner, D. Pachniowski, A. Sandner, M. Ostler, T. Seyller, J. Fabian, M. Ciorga, D. Weiss, and J. Eroms, Phys. Rev. B 87, 081405 (2013).

${ }^{22}$ B. J. Jönsson-Åkerman, R. Escudero, C. Leighton, S. Kim, I. K. Schuller, and D. A. Rabson, Appl. Phys. Lett. 77, 1870 (2000).

${ }^{23}$ T. Maassen, I. Vera-Marun, M. H. D. Guimarães, and B. J. van Wees, Phys. Rev. B 86, 235408 (2012).

${ }^{24}$ C. Ertler, S. Konschuh, M. Gmitra, and J. Fabian, Phys. Rev. B 80, 041405 (2009).

${ }^{25}$ J.-H. Chen, C. Jang, S. Xiao, M. Ishigami, and M. S. Fuhrer, Nat. Nanotechnol. 3, 206 (2008).

${ }^{26}$ W. Han and R. K. Kawakami, Phys. Rev. Lett. 107, 047207 (2011).

${ }^{27}$ A. Dankert, L. Langouche, V. M. Kamalakar, and S. P. Dash, ACS Nano 8, 476 (2014). 